\title{
RIQUEZA Y DIVERSIDAD DE ANFIBIOS Y REPTILES EN UN GRADIENTE ALTITUDINAL EN LA SIERRA DE JUÁREZ, CHIHUAHUA, MÉXICO
}

\section{RICHNESS AND DIVERSITY OF AMPHIBIANS AND REPTILES IN AN ALTITUDINAL GRADIENT AT SIERRA DE JUÁREZ, CHIHUAHUA, MEXICO}

\author{
Antonio Fernández LÓPEZ ${ }^{1, *}$ y Pablo Antonio LAVÍn MURCiO ${ }^{1}$ \\ ${ }^{1}$ Universidad Autónoma de Ciudad Juárez. Instituto de Ciencias Biomédicas. Departamento de Ciencias Químico- \\ Biológicas. Programa de Biología. Laboratorio de Biodiversidad. Anillo Envolvente del PRONAF y Estocolmo \\ s/n. Ciudad Juárez, Chihuahua, México. C.P. 32315 \\ *<antoferlo@gmail.com > (AFL); <plavin@uacj.mx> (PALM) \\ Recibido: 13/01/2015; aceptado: 12/07/2016 \\ Editor responsable: Gustavo Aguirre León
}

Fernández López A. y Lavín Murcio, P. A. (2016). Riqueza y diversidad de anfibios y reptiles en un gradiente altitudinal en la Sierra de Juárez, Chihuahua, México. Acta Zoológica Mexicana (n.s.), 32(3), 230-239.

RESUMEN. El presente estudio herpetofaunístico se realizó de julio del 2004 a julio del 2005 en la Sierra de Juárez, en el norte del estado de Chihuahua. Dicha serranía que se ubica al Oeste de Ciudad Juárez, se encuentra aislada geográficamente, tanto por accidentes naturales como por influencia antropogénica. Los objetivos del proyecto fueron determinar la riqueza y diversidad de los anfibios y reptiles a lo largo de un gradiente altitudinal y con ello contribuir a un mejor entendimiento de su distribución espacial y abundancia temporal. Se registraron 511 individuos de 16 especies, en 52 muestreos realizados. Se describen las condiciones ecológicas y ambientales que revelan la situación actual de esta fauna y su uso potencial como indicadores de disturbio. El conocimiento de la comunidad aquí estudiada es urgente, dada la dinámica que empieza a presentar por las invasiones y modificaciones que se vienen realizando en la Sierra.

Palabras clave: Diversidad herpetológica, Sierra de Juárez, Distribución ecológica.

\section{INTRODUCCIÓN}

Las islas de montaña son terrenos elevados que sobresalen en una secuencia de valles y montañas. Se han detectado alrededor de 20 complejos de islas en todo el mundo (Warshall, 1995). En América del Norte se encuentra el Archipiélago Madrense que se compone de numerosas islas de montaña del sur de Estados Unidos de América y norte de México. Esta región se destaca por presentar una alta diversidad de especies de flora y fauna relacionada con las diferentes comunidades vegetales que se desarrollan a lo largo de los pisos altitudinales, desde ecosistemas
Fernández López A., \& Lavín Murcio, P. A. (2016). Richness and diversity of amphibians and reptiles in an altitudinal gradient at Sierra de Juarez, Chihuahua, Mexico. Acta Zoológica Mexicana (n.s.), 32(3), 230-239.

ABSTRACT. This study was conducted from July 2004 to July 2005 in the Sierra de Juarez, in northern Chihuahua. This mountainous area is located west of Ciudad Juarez, is geographically isolated, by both natural features and anthropogenic influence. The objectives were to determine the richness and diversity of amphibians and reptiles along an altitudinal gradient and thus contribute to a better understanding of their spatial distribution and abundance. Five hundred and eleven individuals of 16 species were recorded in 52 samplings. Ecological and environmental conditions that reveal the current status of this fauna and their potential use as indicators of disturbance are described. Knowledge of our study community is urgent, given the dynamic that is beginning to show by invasions and changes being made in the Sierra.

Key words: Herpetological diversity, Sierra de Juárez, Ecological distribution.

áridos en bajas elevaciones hasta ecosistemas templados en las zonas más altas (Bezy \& Cole, 2014). La Sierra de Juárez y otros grupos de montañas cercanos a ésta, presentan igualmente características de aislamiento desde el Pleistoceno, donde los amplios valles actúan como la principal barrera para la colonización de nuevas especies. Los anfibios y reptiles son sin duda los grupos de fauna que más afecta el aislamiento, pues sus capacidades de dispersión son limitadas en comparación con los mamíferos y las aves. En este contexto, se han realizado diversos estudios de la riqueza de herpetofauna en algunas islas de montaña cercanas a la Sierra de Juárez (González-Flores, 
2005; Martínez-Gómez, 2006; Córdova-Reza, 2007; Astorga, 2007; Chávez, 2007), siendo el más completo el de la región de las Montañas Franklin en El Paso, Texas (Worthington, 1976), donde se registran 63 especies, tanto de zonas de montaña como de valles adyacentes. Los anfibios y reptiles de las zonas desérticas resultan ser excelentes indicadores de los efectos de cambios en ambientes extremos y de la alteración de comunidades en ecosistemas colindantes a urbanizaciones (Oropeza, 2004; Faeth et al., 2005; FAO, 2013). La Sierra de Juárez actualmente presenta problemas de fragmentación y presión de los hábitats naturales por efecto del desarrollo urbano, por lo cual, en el presente proyecto se planteó estimar la riqueza y diversidad de anfibios y reptiles a lo largo de un gradiente altitudinal con el fin de contribuir a un mejor entendimiento de su distribución espacial y abundancia temporal en la Sierra de Juárez, Chihuahua.

\section{MATERIALES Y MÉTODOS}

Área de estudio. La Sierra de Juárez se describe como un macizo montañoso de rocas sedimentarias de tipo marino depositadas durante el período Cretácico (65-144 Ma) (Drewes \& Dyer, 1993), orientado de noroeste a sureste con diversas formaciones topográficas que van desde valles con suelos arenosos o pedregosos hasta amplias elevaciones fuertemente accidentadas, aunque en algunos casos terminan en pequeñas mesetas. Se localiza al suroeste del municipio de Juárez, Chihuahua (Fig. 1). El área de estudio se ubica hacia la parte centro-noroeste del macizo montañoso con elevaciones que van de los 1375 msnm hasta los $1680 \mathrm{msnm}$.

De acuerdo a la nomenclatura de Köppen el clima en la región es muy árido templado $\mathrm{BWk}\left(\mathrm{x}^{\prime}\right)$ con temperatu- ras que pueden situarse por debajo del punto de congelación en invierno, hasta temperaturas máximas de $44^{\circ} \mathrm{C}$ en verano. La precipitación media anual de acuerdo al Servicio Meteorológico Nacional es de $267.5 \mathrm{~mm}$, mismas que suelen ser torrenciales y ocurrir en cualquier época del año. En términos generales, la vegetación se compone de matorral xerófilo que incluye una gran variedad de especies de porte suculento como cactáceas, agaves, yucas y sotoles, además de abundantes hierbas anuales, arbustos y algunos pequeños árboles que pueden alcanzar alturas de tres o cuatro metros como es el caso del encino Quercus turbinella (Green, 1889) y el mezquite Prosopis glandulosa (Torrey, 1827) (Corral \& Worthtington, 1986; Worthington \& Corral, 1987; Goodell, 1997).

Trabajo de campo. Previo a los muestreos formales, se realizó una visita a la Sierra de Juárez para seleccionar el área de estudio definitiva. Se eligió una superficie con un intervalo altitudinal de $355 \mathrm{~m}$ entre el punto más bajo y el de mayor altura. Se definieron cinco sitios de muestreo los cuales presentan algunas características distintivas entre sí, principalmente en términos de la pendiente y composición del terreno, la altitud, la flora dominante y la cercanía a cuerpos de agua (Cuadro 1).

El registro de especies se obtuvo a partir de dos métodos. Uno fue el método directo y consistió en la captura y liberación de los ejemplares (mediante ligas, ganchos herpetológicos y mano desnuda), búsqueda de mudas, así como la visualización y toma de fotografías de los organismos. El nivel del esfuerzo del método directo fue de 520 horas/hombre con un promedio de 104 horas/hombre por sitio de muestreo durante todo el estudio. Para el método indirecto se instalaron 50 trampas de embudo (10/sitio), colocándolas estratégicamente en sitios con al menos algo de sombra para evitar posibles muertes de los ejemplares capturados. También se colocó un trozo de te-

Cuadro 1. Características sobresalientes de los cinco sitios de muestreo en la Sierra de Juárez, Chihuahua. Agle: Agave lechuguilla, Cemo: Cercocarpus montanus, Dawh: Dasylirion wheeleri (S. Watson ex Rothr, 1878), Ecst: Echinocereus stramineus (Engelm.), Epas: Ephedra aspera (Engelm. ex S. Watson, 1882), Fosp: Fouquieria splendens (Engelm., 1879), Latr: Larrea tridentata (Sessé \& Moc. ex DC, 1800), Pain: Parthenium incanum (Kunth), Yutr: Yucca treculeana (Carriére, 1858).

\begin{tabular}{cclccl}
\hline Sitio & $\begin{array}{c}\text { Altitud } \\
(\mathrm{msnm})\end{array}$ & \multicolumn{1}{c}{ Flora dominante } & $\begin{array}{c}\text { Pendiente } \\
(\text { grados })\end{array}$ & $\begin{array}{c}\text { Cuerpos de agua o } \\
\text { corrientes cercanas } \\
(<100 \mathrm{~m})\end{array}$ & Terreno \\
\hline A & 1680 & Fosp, Ecst, Cemo, Agle, Dawh & $6-35$ & NO & Mayormente roca madre \\
B & 1565 & Pain, Latr, Yutr & $10-45$ & NO & Roca madre y suelo pedregoso \\
C & 1450 & Yutr, Agle, Epas, Latr, Pain & $6-25$ & NO & Afloramiento de roca. Mayormente suelo gravoso \\
D & 1395 & Latr, Agle & $0-10$ & SÍ & Mayormente suelo gravoso \\
E & 1375 & Latr, Agle, Fosp & $0-10$ & SÍ & Suelo medianamente gravoso y arenoso \\
\hline
\end{tabular}




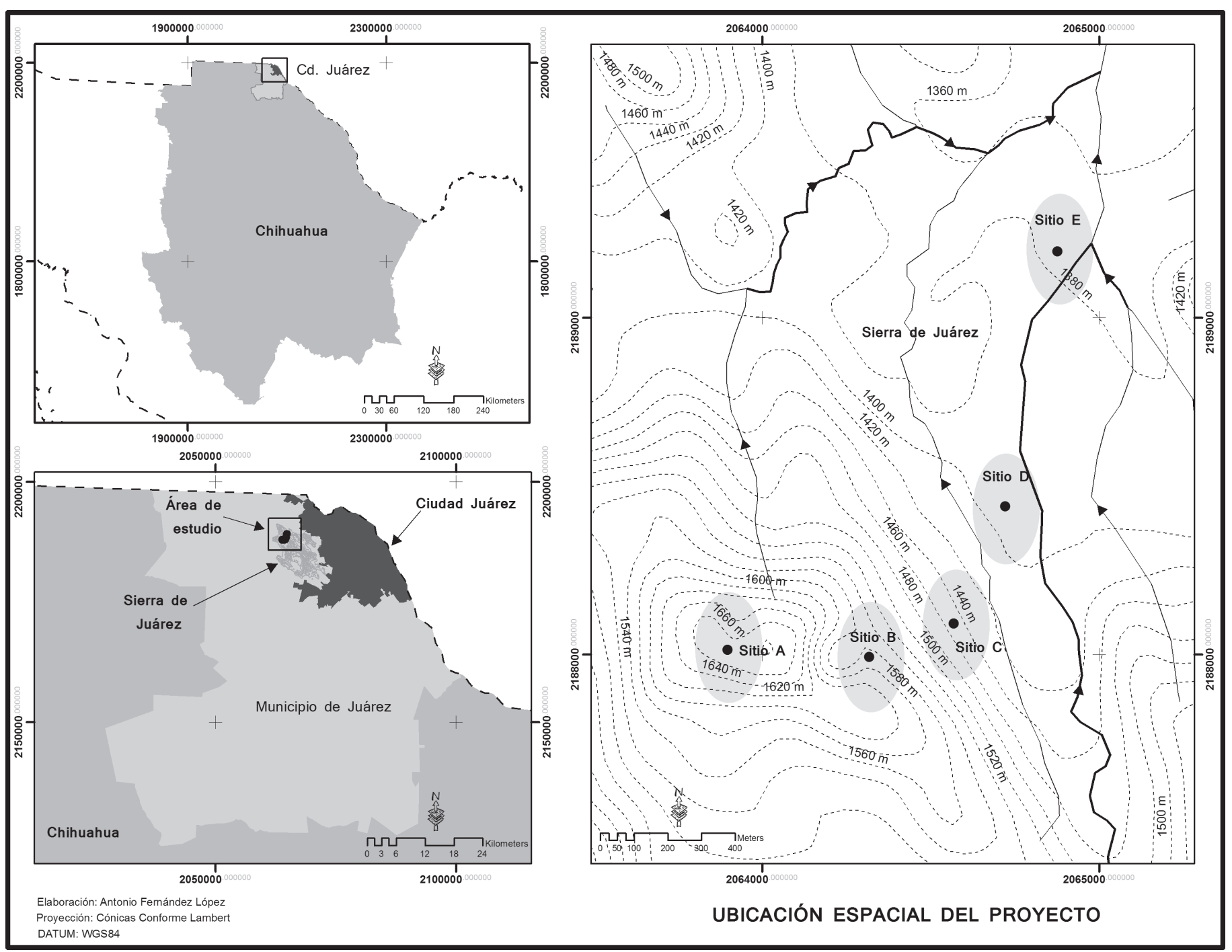

Figura 1. Ubicación macroespacial y específica de los sitios de muestreo en la Sierra de Juárez, municipio de Juárez, Chihuahua.

la de yute sobre cada trampa y se puso un tubo de PVC en el interior para aminorar el calor dentro de la trampa. Las trampas permanecieron abiertas aproximadamente 624 horas durante todo el estudio, lo que da un promedio de 12 horas por muestreo (por día). Dado que los muestreos se realizaban en promedio cada quince días durante dos días consecutivos (dos muestreos) las trampas permanecían abiertas prácticamente 24 horas seguidas, y al término del segundo muestreo quincenal se cerraban para evitar muertes innecesarias hasta la realización de los próximos muestreos.

Se realizaron 52 muestreos diurnos de julio del 2004 a julio del 2005 con un promedio de cuatro salidas al mes, revisando las trampas al tiempo que se muestreaba de for- ma directa. Todos los ejemplares fueron contabilizados con el fin de estimar su abundancia según los criterios de Cox (1990) y Padilla (1996) que indican como especie rara $(\mathrm{R})$ aquella con registros de uno o dos ejemplares; especie moderadamente abundante (MA) aquella de tres a cinco registros; y especie abundante $(\mathrm{AB})$ aquella con seis o más registros. La identificación de las especies se realizó a través del uso de literatura especializada (FloresVillela et al., 1995; Degenhardt et al., 1996; Campbell \& Lamar, 2004; Lemos \& Smith, 2009). Finalmente se hizo mención sobre las especies referidas bajo algún estatus de riesgo de acuerdo a la NOM-059-SEMARNAT-2010.

Análisis de datos. La diversidad alfa se midió como el número de especies en cada sitio de muestreo. Estos se 
compararon en términos de su abundancia, composición y uniformidad a través de gráficas rango-abundancia (Carvajal-Cogollo, \& Urbina-Cardona, 2008). Por otro lado, se realizó una curva de acumulación de especies para el total de los sitios a lo largo de los muestreos y se aplicaron los estimadores de riqueza Chao 2 e ICE, además de graficarse las especies únicas (singletons) y las duplicadas (doubletons) para ver si decrecían a medida que aumentaban los muestreos (Moreno, 2001; Villarreal et al., 2004); dichos cálculos se realizaron con el programa EstimateS versión 8.2 (Colwell, 2006).

La diversidad herpetológica de los sitios de muestreo se estimó a través del índice de Shannon-Wiener. Este expresa la uniformidad de los valores de importancia a través de todas las especies de la muestra.

Para determinar la diversidad beta se utilizó el índice de complementariedad propuesto por Colwell \& Coddington (1994). Los valores pueden oscilar entre cero (cuando ambos sitios se componen por las mismas especies) y 1 (cuando la composición de especies es completamente distinta).

\section{RESULTADOS}

Se registraron un total de 511 individuos divididos en dos especies de anfibios y 14 de reptiles. Los anfibios comprendieron el $29.7 \%$ del total registrado, es decir, 152 ejemplares. Para los reptiles se obtuvo el $70.3 \%$ de los registros equivalentes a 359 individuos. De estos últimos, se identificaron ocho especies de lagartijas con 347 ejemplares, mientras que para las serpientes solo se obtuvieron 12 registros de seis especies (Cuadro 2).

A nivel de los sitios de muestreo, para el sitio A se registraron solo cinco ejemplares de dos especies de lagartijas; el sitio B incluyó un total de 22 individuos de un anfibio y seis reptiles; el sitio C presentó 31 individuos de un anfibio y seis reptiles; para el sitio D se registraron 141 ejemplares de dos especies de anfibios y nueve reptiles; y finalmente para el sitio E se obtuvo un total de 312 ejemplares de dos especies de anfibios y nueve reptiles.

Las especies más abundantes fueron Anaxyrus punctatus, Cophosaurus texanus, Aspidoscelis exsanguis y Aspidoscelis marmorata con 131, 110, 107 y 69 individuos respectivamente. También se registraron algunas especies catalogadas como raras, principalmente para el grupo de las serpientes como fueron Crotalus lepidus, Sonora semiannulata y Pituophis catenifer por mencionar algunas.
Se registraron cinco especies referidas en la NOM059-SEMARNAT-2010, las cuales abarcan el 31.2\% del total registrado. Dos se encuentran Sujetas a Protección Especial (Pr) y tres bajo el estatus de amenazadas (A).

Los estimadores de riqueza ICE y Chao 2 mostraron valores ligeramente variables entre los diferentes sitios de muestreo. Para el sitio A el valor observado es prácticamente el mismo al de los estimadores; en el sitio B se registraron siete especies, mientras que los estimadores Chao 2 e ICE sugieren una riqueza de 10 y 17 especies respectivamente; en el sitio $\mathrm{C}$ se registraron siete especies con valores de Chao 2 e ICE de 7 y 8 respectivamente; en el sitio D la riqueza observada fue de 11 especies, mientras que los estimadores Chao 2 e ICE indican riquezas de 11 y 12 especies para cada cual; y finalmente la riqueza de especies observada para el sitio E fue de $11 \mathrm{y}$ para sus correspondientes estimadores se obtuvieron valores de 12 y 14 especies respectivamente. Básicamente en la mayoría de los sitios de muestreo los valores únicos (singletons) se mantuvieron estables o ligeramente a la baja, mientras que los duplicados (dobletons) mostraron un incremento ligero, pero evidente, principalmente para el sitio C (Fig. 2).

Por su parte, el índice de Shannon mostró el valor más bajo para el sitio A con $\mathrm{H}^{\prime}=0.67$, seguido por el sitio B con $H=$ '1.42. Los sitios $C$, D y E obtuvieron los valores relativamente más altos con valores de $\mathrm{H}^{\prime}$ de $1.63,1.69$ y 1.65 respectivamente (Fig. 3).

El índice de complementariedad arrojó valores diversos de igualdad y diferencia. El valor de mayor igualdad corresponde a los sitios D y E con 0.30, mientras que los sitios de mayor diferencia son A-D y A-E con un valor de 0.81 para ambas combinaciones (Cuadro 3).

Las gráficas de rango-abundancia muestran resultados variables entre uno y otros sitios, lo que indica que los patrones de similitud en la distribución y abundancia de las especies dominantes y raras es igualmente variable entre ellos (Fig. 4). Para casi todos los sitios las especies claramente dominantes fueron las lagartijas Crotaphytus collaris y Aspidoscelis exsanguis, manteniéndose posicionadas en el punto más alto a lo largo del gradiente altitudinal.

\section{DISCUSIÓN}

El total de especies registradas para la Sierra de Juárez resulta una importante contribución al conocimiento de la riqueza herpetológica de la zona. Comparativamente 
Fernández-López \& Lavín-Murcio et al.: Anfibios y reptiles de la Sierra de Juárez, Chihuahua

Cuadro 2. Listado de anfibios y reptiles registrados en Sierra de Juárez, Chihuahua, abundancias por sitio de muestreo, código para las especies y estatus de riesgo según la NOM-059-SEMARNAT-2010. A: Amenazada; Pr: Sujeta a Protección Especial.

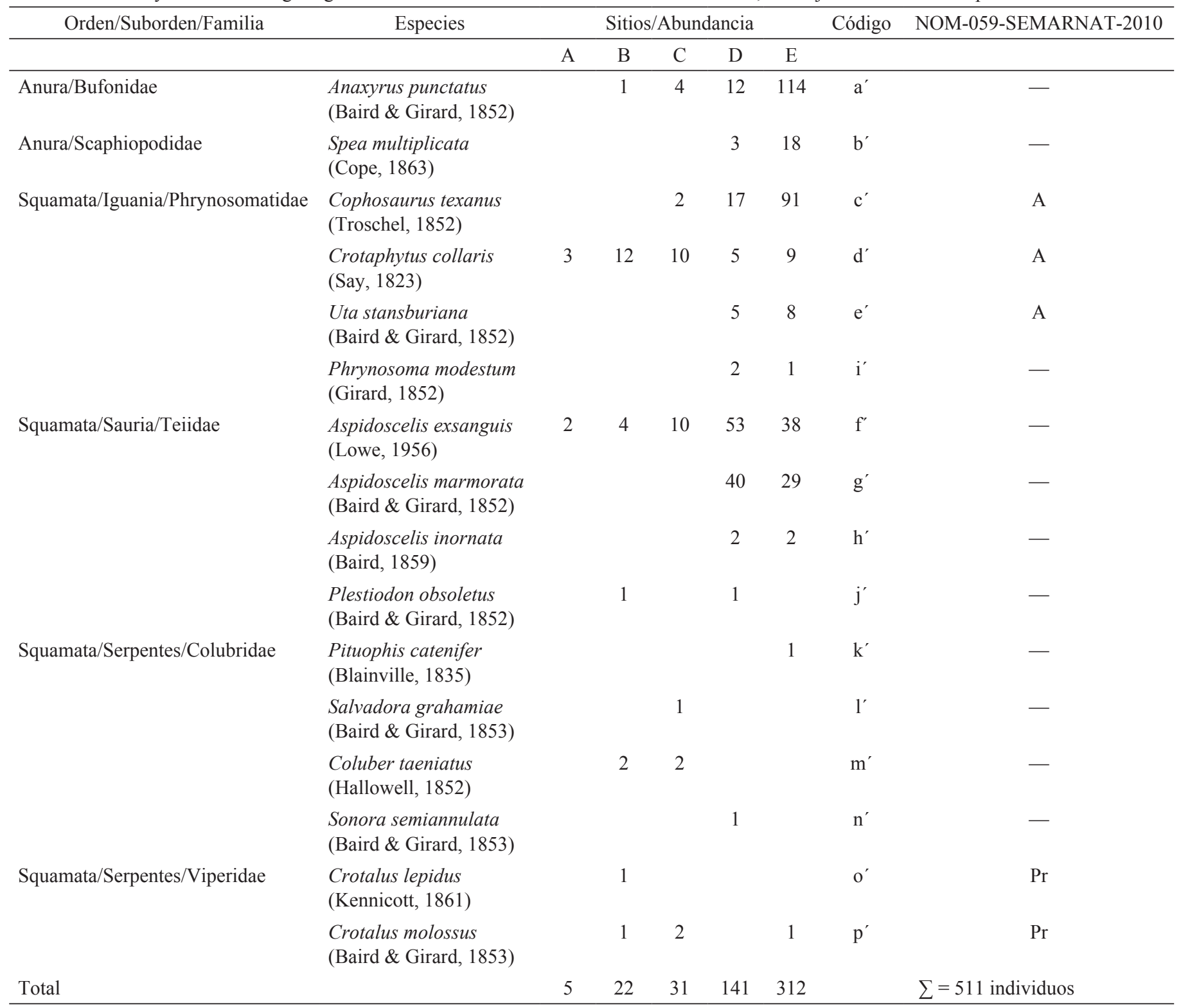

con otros macizos montañosos aislados que se encuentran relativamente cerca de la zona de estudio, se han registrado riquezas de especies similares hacia la zona de los municipios de Janos (23 especies), Nuevo Casas Grandes (20 especies) y Ahumada (26 especies) (González-Flores, 2005; Martínez-Gómez, 2006; Astorga, 2007; Chávez, 2007; Córdova-Reza, 2007). No obstante, en las Islas de Montaña correspondientes a Estados Unidos de América, se han realizado estudios importantes sobre la riqueza de herpetofauna, registrándose altas riquezas, tanto en Arizona y New Mexico, como en Texas. Bezy \& Cole (2014) atribuyen una alta diversidad de anfibios y reptiles en el Archipiélago Madrense de Arizona y New Mexico debido a la variedad de ecosistemas (bosques y chaparral) y a las condiciones ambientales más favorables que en áreas desérticas. Por su parte, Worthington (1976) registró un total de 63 especies de herpetofauna para la región de las Montañas Franklin, donde las condiciones de los ecosistemas son muy similares a la Sierra de Juárez, aunque cabe destacar que una parte considerable de las especies registradas solo se distribuyen en ambientes riparios o lacustres (perennes), fuera de las tierras serranas. 


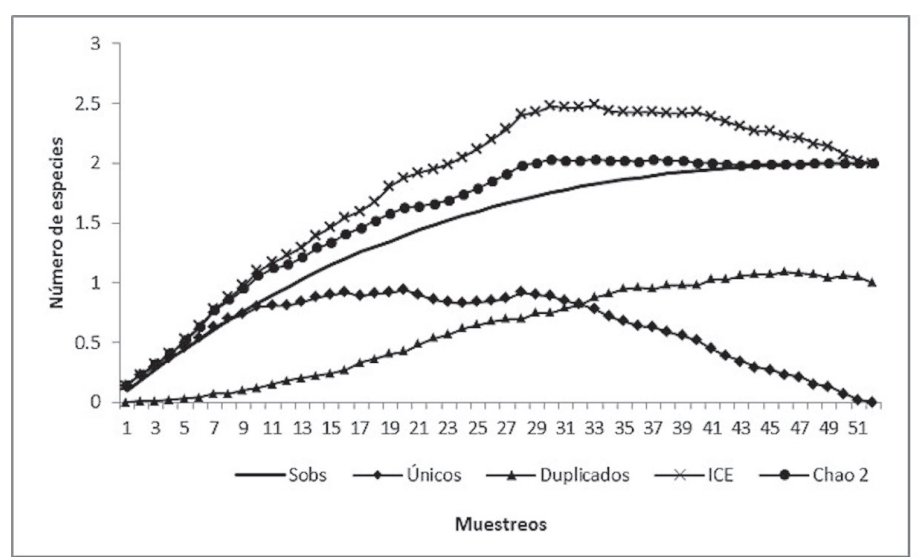

Sitio A

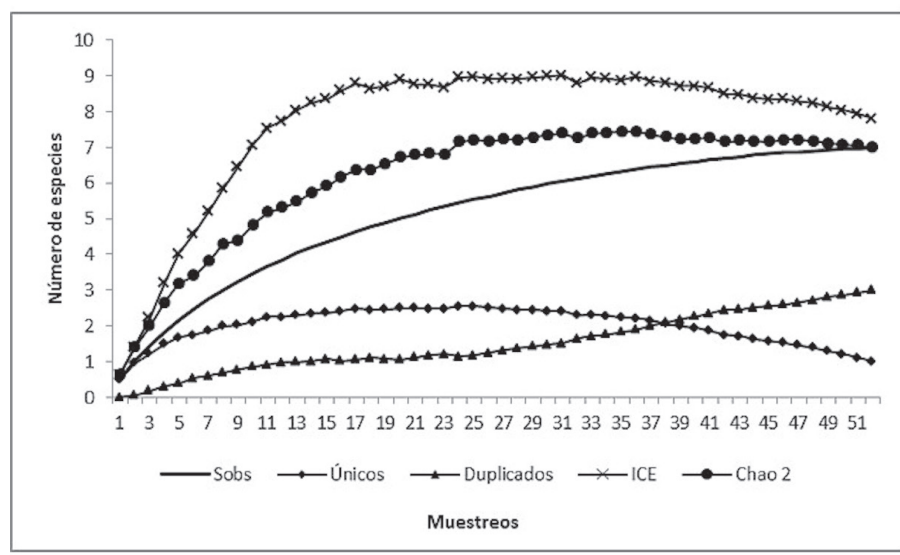

Sitio C

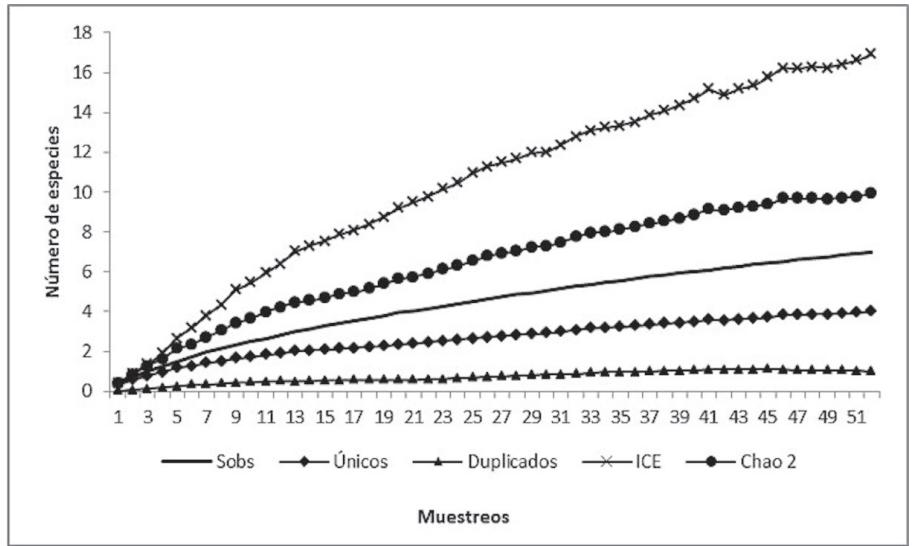

Sitio B

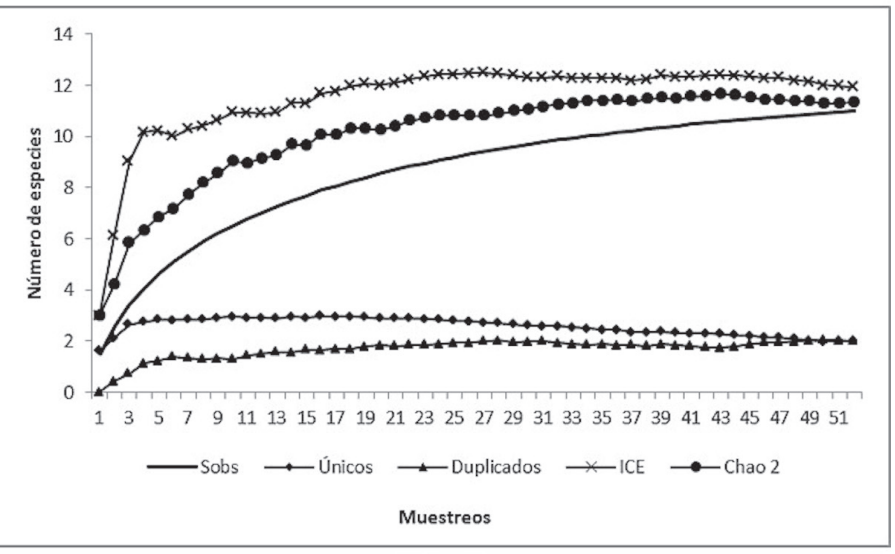

Sitio D

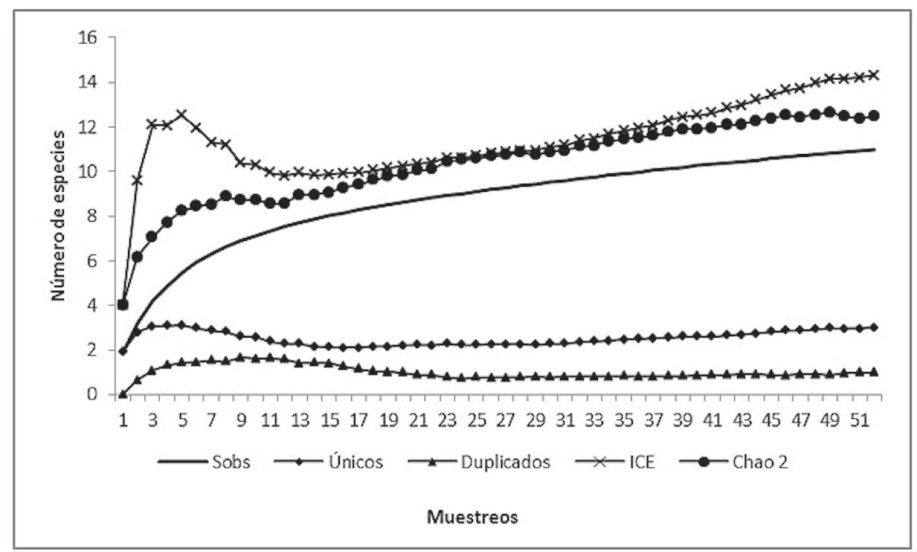

Sitio $\mathrm{E}$

Figura 2. Curvas de acumulación de especies de herpetofauna para los cinco sitios de muestreos en la Sierra de Juárez.

Cabe mencionar con fines únicamente informativos y no para el análisis de los resultados que aquí se exponen, que se registraron otras tres especies de reptiles hacia la parte sur y extremo norte de la Sierra, es decir, fuera de los sitios de muestreo en tiempo y espacio, pero que son importantes como adhesiones a la riqueza herpetológica de la Sierra de Juárez (Cuadro 2). Las especies encontradas en la parte sur son Crotalus atrox (Baird \& Girard, 


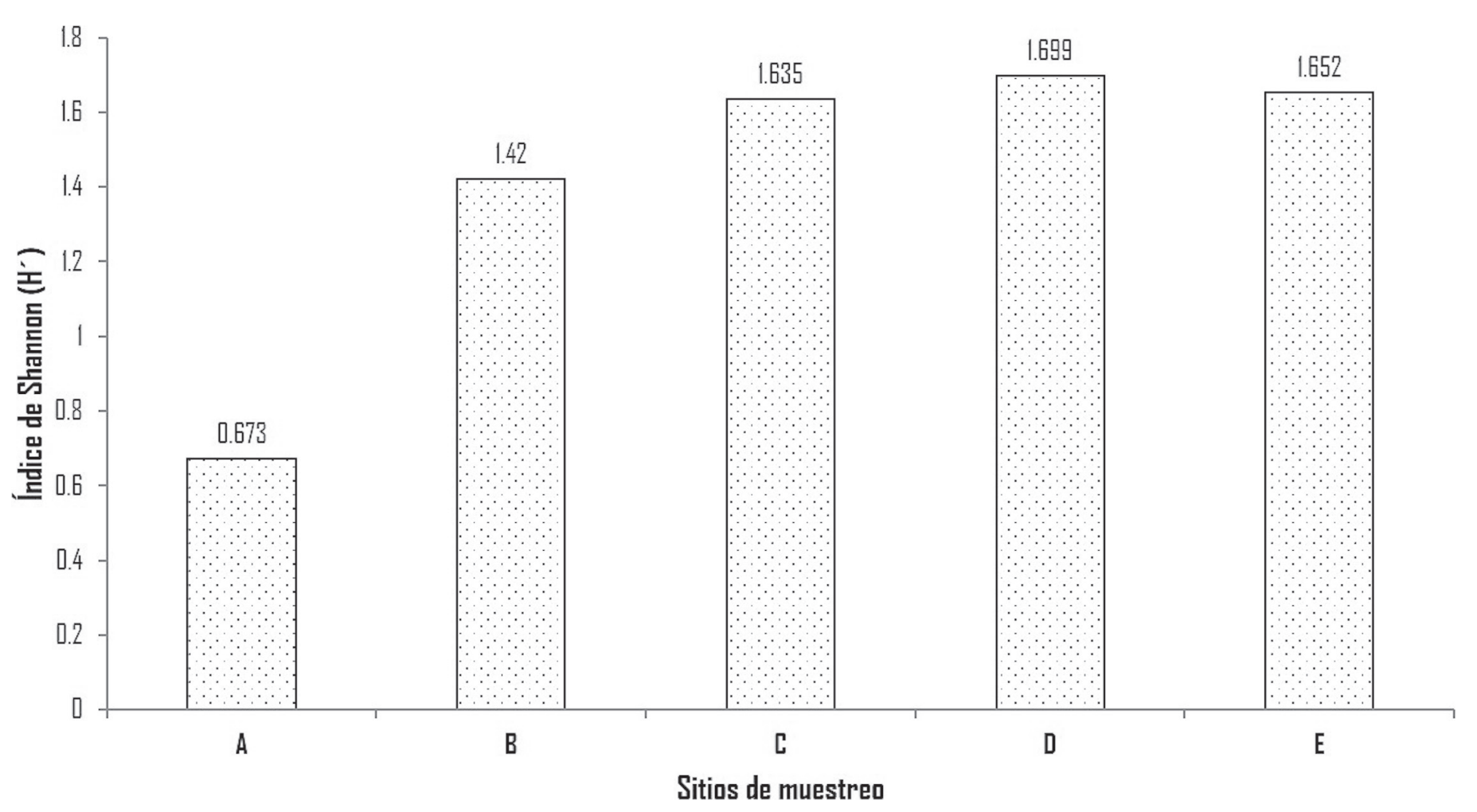

Figura 3. Valores del Índice de Shannon de la herpetofauna en los sitios de muestreo en la Sierra de Juárez.

1853) y Phrynosoma cornutum (Harlan, 1824). La especie registrada en la parte norte de la Sierra es Sceloporus magister (Hallowell, 1854).

Si bien la Sierra de Juárez presenta uno de los ambientes más secos en relación con otras sierras aisladas, el registro de 511 individuos de anfibios y reptiles sugiere una importante abundancia que denota que a pesar de las condiciones ambientales extremas de la región, existen especies con poblaciones numerosas, tales como Anaxyrus punctatus, Cophosaurus texanus, Aspidoscelis exsanguis y Aspidoscelis marmorata de los cuales se registraron

Cuadro 3. Valores de complementariedad (Colwell \& Coddington, 1994) para los cinco sitios de muestreo en Sierra de Juárez, Chihuahua.

\begin{tabular}{cccccc}
\hline $\begin{array}{c}\text { Sitios de } \\
\text { muestreo }\end{array}$ & A & B & C & D & E \\
\hline A & 0 & 0.71 & 0.71 & 0.81 & 0.81 \\
B & --- & 0 & 0.44 & 0.71 & 0.71 \\
C & --- & --- & 0 & 0.71 & 0.61 \\
D & --- & --- & --- & 0 & 0.30 \\
E & --- & --- & --- & --- & 0 \\
\hline
\end{tabular}

más de 60 individuos para cada especie a lo largo de los muestreos. Este hecho las califica como especies abundantes de acuerdo a Cox (1990) y Padilla (1996), en contraste con especies que resultaron raras, ya por sus hábitos $\mathrm{y}$ conductas o porque naturalmente sus poblaciones son mucho más reducidas en comparación con las especies antes mencionadas. En este grupo de especies raras se pueden mencionar las serpientes Crotalus lepidus, Sonora semiannulata y Pituophis catenifer.

Es preciso señalar que la Sierra de Juárez, a diferencia de otras islas de montaña, se encuentra significativamente aislada de otros macizos montañosos desde el Pleistoceno y rodeada por sistemas de dunas que han constituido una barrera infranqueable para muchas especies. Además, los vestigios de vegetación templada son muy reducidos, pero a pesar de lo anterior y de la reciente expansión urbana, mantiene poblaciones de especies de hábitats mésicos como Crotalus molossus y Crotalus lepidus que requerirán una atención especial.

La riqueza de especies también se reflejó de manera muy puntual entre sitios de muestreo sobresaliendo los sitios E y D con el mayor número de especies (11) y con la mayor abundancia de ejemplares registrados (312 y 

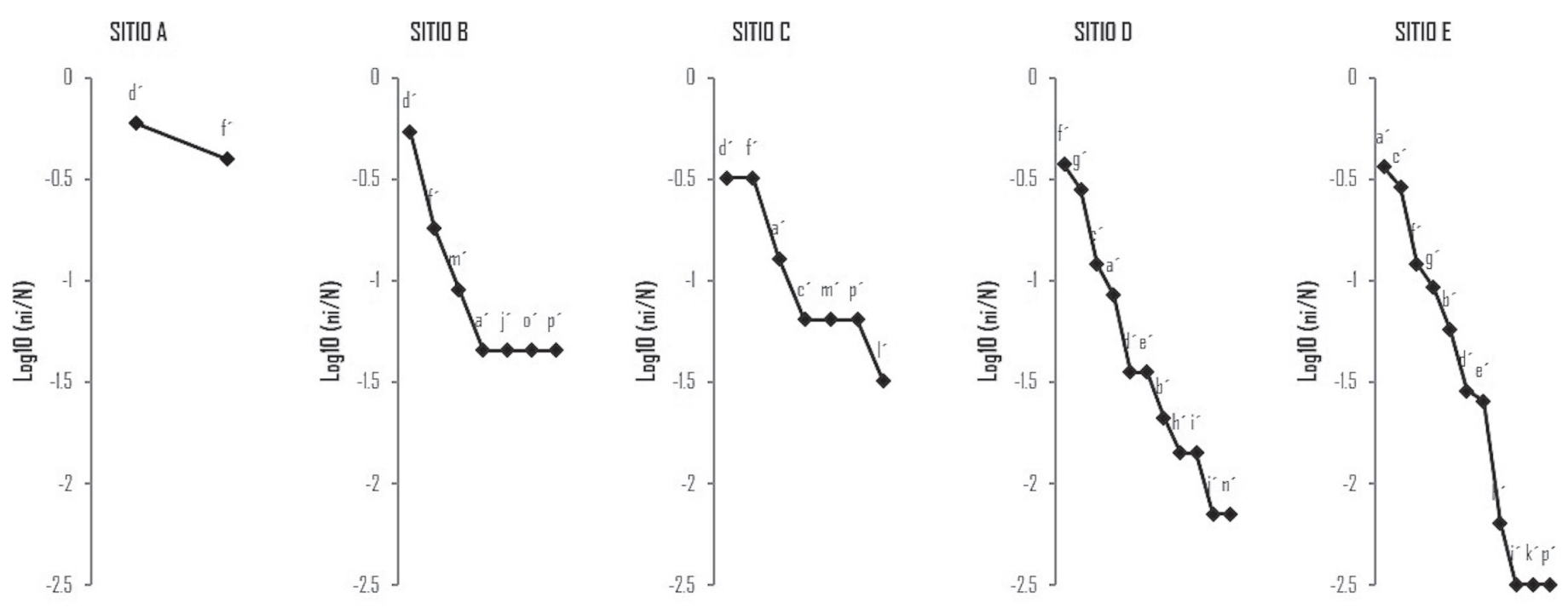

Rango abundancia de las especies

Figura 4. Gráficas de rango-abundancia para la herpetofauna registrada en los cinco sitios de muestreo. Los códigos de las especies se muestran en el cuadro 2.

141 respectivamente). Este hecho podría atribuirse a la presencia de un cuerpo de agua temporal y a la cercanía de un río intermitente, que proveen en cierta medida, ambientes más favorables para la presencia de herpetofauna en términos de riqueza y abundancia de especies (González et al., 2006; Pedroza \& Angarita, 2011). El sitio con el menor número de especies corresponde al sitio A (apenas con cinco ejemplares de dos especies), mismo que se encuentra en la parte más alta de uno de los cerros de la Sierra con un suelo mayormente expuesto a la meteorización, niveles más altos de radiación solar y una muy baja cubierta vegetal, propiciando condiciones ambientales poco favorables para el establecimiento de anfibios y reptiles (Bezy \& Cole, 2014).

La aplicación de los estimadores de riqueza destacó a Chao 2 como el más acertado de acuerdo a los registros obtenidos, donde en los sitios A, C y D las curvas de acumulación de especies se emparejan hacia la parte final, y solo en los sitios B y E se esperan dos y una especies por encontrar respectivamente. Este hecho sugiere un buen nivel de muestreo al haberse detectado, de manera general, cerca del $100 \%$ de las especies esperadas. Para el caso de ICE, en tres de los sitios de muestreo (A, C y D) se obtuvo un buen nivel de muestreo según las especies esperadas (>90\%). Únicamente para los sitios B y $\mathrm{E}$ las especies esperadas que indica el estimador ICE se elevan considerablemente, al grado de solo haber encontrado el $41 \%$ y $78 \%$ de las especies esperadas respectivamente. Por su parte, las especies únicas y duplicadas mostraron un comportamiento estable en términos generales, sobresaliendo en cierta medida para el sitio $\mathrm{C}$ las especies duplicadas, con una ligera tendencia de ascenso a lo largo de los muestreos. Sin duda las condiciones climáticas influyeron fuertemente en los resultados obtenidos, ya que las altas y bajas temperaturas, además de la baja humedad ambiental, no permitieron registrar otras especies, principalmente raras como serpientes, que seguramente comparte la Sierra de Juárez con las Montañas Franklin, a pesar de haber instalado trampas de embudo, las cuales resultaron menos efectivas en comparación con el método de captura directa, es decir, con las trampas se logró registrar un total de nueve especies del total de 16, mientras que de forma directa se registraron 15 de las 16 especies totales. En este sentido, un aporte interesante que se puede destacar del uso de las trampas de embudo en el presente estudio, es la captura de Plestiodon obsoletus. A este respecto, una modificación en los horarios de muestreo (crepuscular y nocturno) y la implementación de sistemas de trampeo (trampas de caída y cercas de desvío) podrían aumentar la probabilidad de nuevos registros, principalmente de especies esquivas o raras. 
La diversidad herpetológica de acuerdo con el Índice de Shannon (Fig. 3) mostró que el sitio A representa el menos diverso $\left(\mathrm{H}^{\prime}=0.67\right)$. Esto está en concordancia con las características propias del sitio, al estar expuesto mayormente al intemperismo, proveyendo menores condiciones para el establecimiento de la herpetofauna. Por su parte, los sitios C, D y E alcanzaron valores más aceptables en función de las condiciones ambientales de los sitios, sobre todo por la presencia de un cuerpo de agua temporal relativamente cercano a los sitios $\mathrm{E}$ y $\mathrm{D}$, mientras que el sitio $\mathrm{C}$ se ubica a una distancia ligeramente superior a 100 metros, tanto del cuerpo de agua como de uno de los ríos temporales más importantes de la Sierra, por lo que aún debe existir cierta influencia de las condiciones con el sitio en cuestión. Los valores obtenidos de $\mathrm{H}^{\prime}$ se encuentran entre 1.63 y 1.69 .

Al comparar los sitios a través del índice de complementariedad, los sitios D-E presentaron mayor similitud entre sí, con un valor de 0.30 , mostrando que se trata de sitios muy parecidos en lo que a su composición herpetológica se refiere (Cuadro 3). Por su lado, la combinación A-D y A-E, resultaron como aquellos con menor similitud al alcanzar un valor para ambas combinaciones de 0.81 , siendo éste bastante alto, lo que demuestra que la composición de especies es muy distinta para el sitio A en relación a los sitios D y E. De hecho las especies encontradas para el sitio A (Crotaphytus collaris y Aspidoscelis exsanguis) son las únicas que se encontraron distribuidas en todo el intervalo altitudinal, destacándolas como especies con una importante adaptación a hábitats extremos con diferentes características ambientales (Hammerson et al., 2007; Lemos-Espinal \& Smith, 2009).

En este contexto, lo antes mencionado se puede corroborar a través de las gráficas de rango- abundancia (Fig. 4), donde claramente $C$. collaris y $A$. exsanguis denotan una dominancia en los sitios A, B y C. En el sitio D $A$. exsanguis se mantuvo a la cabeza, mientras que C. collaris se ubicó en una posición media. Para el sitio E ambas especies se localizaron hacia la parte media-alta de la gráfica. Para el resto de las especies, los valores, y por ende su posición en las gráficas, fueron muy variables. A su vez, la composición de especies entre sitios de muestreo resultó muy diferente en la mayoría de los casos, siendo el más similar la combinación D-E. Por otro lado, la distribución de las abundancias igualmente mostró diferencias importantes en casi todos los sitios, excepto nuevamente los sitios D-E. Esta situación puede atribuirse a que las condiciones ambientales, como ya se ha dicho antes, son relativamente similares para dichos sitios de muestreo.
Frente a los resultados encontrados, la importancia de conservar los hábitats naturales de la Sierra de Juárez es un hecho de gran relevancia ante el imparable crecimiento urbano y la presión que constantemente se ejerce sobre los recursos naturales (Faeth et al., 2005). Aunado a esto, de las especies registradas, destaca la presencia de cinco de ellas como referidas en la NOM-059-SEMARNAT-2010, tres bajo el estatus de Amenazadas (A) y dos Sujetas a Protección Especial (Pr). Su importancia radica, en que en suma, ocupan un porcentaje alto de la herpetofauna de la Sierra de Juárez (31.2\%), fungiendo su calidad de riesgo, como un elemento para la implementación de estrategias de conservación de la Sierra y su fauna en un futuro cercano, más aún, al haber actualmente planes de expansión de la mancha urbana en sus laderas occidentales. Lo anterior significaría un aislamiento total de las poblaciones de herpetofauna y un incremento en el riesgo de extinción local para algunas especies.

AGRADECIMIENTOS. A Nicolás Urbina Cardona por sus valiosos $\mathrm{y}$ bien atinados comentarios al presente manuscrito.

\section{LITERATURA CITADA}

Astorga, M. I. (2007). Herpetofauna de la Sierra Peñascos, municipio de Ahumada, Chihuahua, México. Tesis de licenciatura, Universidad Autónoma de Ciudad Juárez.

Bezy, R. L., \& Cole, C. J. (2014). Amphibians and reptiles of the Madrean Archipelago of Arizona and New Mexico. American Museum Novitates, 3810, 1-24.

Campbell, J. A., \& Lamar, W. W. (2004). The Venomous Reptiles of the Western Hemisphere. Volume II. Comstock Publishing Associates. New York, 870 pp.

Chávez, G. (2007). Reptiles de la Sierra La Candelaria, municipio de Ahumada, Chihuahua, México. Tesis de licenciatura, Universidad Autónoma de Ciudad Juárez.

Carvajal-Cogollo, J. E., \& Urbina-Cardona, J. N. (2008). Patrones de diversidad y composición de reptiles en fragmentos de bosque seco tropical en Córdoba, Colombia. Tropical Conservation Science, 1(4), 397-416.

Colwell, R. K. (2006). EstimateS: statistical estimation of species richness and shared species from samples. (http://viceroy.eeb. uconn.edu/estimates) (Acceso enero 2012) Version 8. Persistent URL<purl.oclc.org/estimates>.

Colwell, R. K., \& Coddington, J. A. (1994). Estimating terrestrial biodiversity through extrapolation. Philosophical Transactions of the Royal Society of London Series B, 345, 101-118.

Córdova-Reza, J. N. (2007). Ofidios de la Sierra "La Escondida" en Nuevo Casas Grandes, Chihuahua. Tesis de licenciatura. Universidad Autónoma de Ciudad Juárez.

Corral, R. D., \& Worthington, R. D. (1986). A Survey of Winter Annuals in the Sierra de Juárez, Chihuahua, México, for effects of 
El Paso-Juárez Pollution. The Journal of the Colorado-Wyoming Academy of Sciences, XVIII 1, 1-7.

Cox, W. (1990). Laboratory Manual of General Ecology. William C. Brown Editions. Dubuque, Iowa, 251 pp.

Degenhardt, W. G., Painter, C. W., \& Price, A. H. (1996). Amphibians and Reptiles of New Mexico. University of New Mexico, Alburquerque, $431 \mathrm{pp}$.

Drewes, H., \& Dyer, R. (1993). Tertiary and Quaternary Structure and Paleotectonics of the Hueco Basin, Trans-Pecos Texas and Chihuahua, Mexico. Bureau of Economic Geology.

Faeth, S. H., Warren, P. S., Shochat, E., \& Marussich, W. A. (2005). Trophic dynamics in Urban Communities. BioScience, 55(5), 399-407.

FAO. (2013). La fauna silvestre en un clima cambiante. Organización de las Naciones Unidas para la Agricultura y la Alimentación, Roma, 119 pp.

Flores-Villela O., Mendoza, Q. F., \& González, P. G. (1995). Recopilación de claves para la determinación de anfibios y reptiles de México. Publicaciones. Especiales Museo de Zoología, 10, 1 285.

Gonzales, L., Muñoz, A., \& Cortez, E. (2006). Primer reporte sobre la herpetofauna de la Reserva Natural "El Corbalán", Tarija, Bolivia. Kempffiana, 2(1), 72-94.

González-Flores, B. G. (2005). Contribución al conocimiento de los ofidios y los anuros de la Sierra de Enmedio, municipio de Janos, Chihuahua, México. Tesis de licenciatura. Universidad Autónoma de Ciudad Juárez.

Goodell, P. C. (1997). Sierra de Juárez: Environmental Park Development. A Proposal. UTEP, El Paso Texas.

Hammerson, G. A., Frost, D. R., \& Santos-Barrera, G. (2007). Aspidoscelis exanguis. The IUCN Red List of Threatened Species 2007. e.T64264A12751408. (acceso junio 2016) http://dx.doi. org/10.2305/IUCN.UK.2007.RLTS.T64264A12751408.en

Lemos-Espinal, J. A., \& Smith, H. M. (2009). Anfibios y reptiles del estado de Chihuahua, México. UNAM, CONABIO. México, $613 \mathrm{pp}$.

Martínez-Gómez, D. A. (2006). Listado de anuros y larcetilios de la Sierra La Escondida, Nuevo Casas Grandes, Chihuahua. Tesis de licenciatura. Universidad Autónoma de Ciudad Juárez.

Moreno, C. E. (2001). Métodos para medir la biodiversidad. Vol. I. M\&T-Manuales y Tesis SEA, Zaragoza, 84 pp.
Oropeza, O. O. (2004). Evaluación de la vulnerabilidad a la desertificación, pp. 303-313. In: Martínez J. \& Fernández L. Cambio climático: Una visión desde México. Secretaria de Medio Ambiente y Recursos Naturales. Instituto Nacional de Ecología, México.

Padilla, G. U. (1996). Distribución herpetofaunística del estado de Querétaro en un gradiente altitudinal y de vegetación. Tesis de licenciatura. Universidad Autónoma de Querétaro.

Pedroza, B. R., \& Angarita, S. T. (2011). Herpetofauna de los humedales La Bolsa y Charco de Oro, Andalucía, Valle del Cauca, Colombia. Revista de la Academia Colombiana de Ciencias, 135, 243-260.

SEMARNAT [Secretaría del Medio Ambiente y Recursos Naturales]. (2010). Norma Oficial Mexicana NOM-059-SEMARNAT2010, Protección ambiental-Especies nativas de México de flora y fauna silvestres: Categorías de riesgo y especificaciones para su inclusión, exclusión o cambio-Lista de especies en riesgo. Diario Oficial de la Federación. 2da Sección, 30 de diciembre de 2010.

Villarreal, H., Álvarez, M., Córdoba, S., Escobar, F., Fagua, G., Gast, F., Mendoza, H., Ospina, M., \& Umaña, A. M. (2004). Manual de métodos para el desarrollo de inventarios de biodiversidad. Programa de Inventarios de Biodiversidad. Instituto de Investigación de Recursos Biológicos Alexander von Humboldt, Bogotá, Colombia, 236 pp.

Warshall, P. (1995). The Madrean Sky Island Archipelago: A planetary overview, pp. 6-18. In: DeBano, L. F., P. F. Ffolliott, A. Ortega-Rubio, G. Gottfried, R. H. Hamre, C. B. Edminster (Tech. Coord.). Biodiversity and management of the Madrean Archipelago: the Sky Islands of Southwestern United States and Northwestern Mexico. Technical Report RM-GTR-264. U.S. Dept. of Agriculture, U.S. Forest Service, Ft. Collins, CO.

Worthington, R. D. (1976). Herpetofauna of the Franklin Mountains, El Paso County, Texas. El Paso Geological Society Symposium on the Franklin Mountains. Quinn Memorial Volume.

Worthington, R. D., \& Corral, R. D. (1987). Some effects of fire on shrubs and succulents in a Chihuahuan Desert Community in the Franklin Mountains, El Paso Country, Texas, pp. 3-17. In: Contributed Papers of the Second Symposium on Resources of the Chihuahuan Desert Region, United States and México. The Chihuahuan Desert Research Institute, Alpine, Texas. 